\title{
An Observational Study on the Effect of Nimodipine on Cerebral Blood Flow Velocity and Oxygenation in Patients with Subarachnoid Haemorrhage
}

\author{
Ruby Chackupurakal ${ }^{1}$, Marlene Gebauer ${ }^{1}$, Fabian Dusse ${ }^{1,2}$, Alexander Hartmann ${ }^{3}$, Makoto Nakamura ${ }^{3}$, \\ Frank Wappler ${ }^{1}$, Samir G Sakka ${ }^{1,4}$ \\ ${ }^{1}$ Department of Anaesthesiology and Operative Intensive Care Medicine, Medical Center Cologne Merheim, University of Witten/Herdecke, Cologne, \\ Germany \\ ${ }^{2}$ Department of Anaesthesiology and Operative Intensive Care Medicine, University Hospital of Cologne, Cologne, Germany \\ ${ }^{3}$ Department of Neurosurgery, Medical Center Cologne Merheim, University of Witten/ Herdecke, Cologne, Germany \\ ${ }^{4}$ Department of Intensive Care Medicine, Medical Center Mittelrhein, Koblenz, University of Mainz, Koblenz, Germany
}

Received: October 30, 2020

Accepted: November 19, 2020

Corresponding Author:

Ruby Chackupurakal M.D.

Department of Anaesthesiology and Operative Intensive Care Medicine Medical Center Cologne Merheim University of Witten/Herdecke Ostmerheimer Str. 200 D-51109 Köln, Germany Tel: +49-221-8907-13814

Fax: +49-221-8907-8541

E-mail: ChackupurakalR@

kliniken-koeln.de

This work has been presented in part at the national Conference of Anaesthesia and Intensive Care Medicine 2015 and 2018 (Hauptstadtkongress der DGAI für Anästhesiologie und Intensivtherapie).

\section{Objective}

In patients with subarachnoid haemorrhage (SAH), nimodipine is administered to prevent for cerebral vasospasm. In this prospective observational study, we examined the effect of nimodipine on cerebral blood flow velocity (BFV) (transcranial Doppler, TCD) and regional oxygen saturation $\left(\mathrm{rSO}_{2}\right)$ (near-infrared spectroscopy, NIRS) as well as its systemic haemodynamic effects.

\section{Methods}

After ethics approval, we enrolled 18 adult patients ( 11 females) with SAH after aneurysm rupture. After treatment of the bleeding source, patients underwent extended haemodynamic monitoring by transpulmonary thermodilution (PiCCOTM, Pulsion, Maquet Getinge Group, Feldkirchen, Germany). Measurements of systemic haemodynamics as well as cerebral oxygenation and blood flow velocity were performed 30 minutes before and after the enteral administration of nimodipine $(60 \mathrm{mg})$. Data was compared by paired t-test. A p $<0.05$ was considered as statistically significant. Data are expressed as mean $\pm \mathrm{SD}$.

Results

Patients' mean age was $59 \pm 11$ years. Enteral nimodipinincrease in heart rate $(75 \mathrm{vs.} 79 / \mathrm{min}, \mathrm{p}<0.05)$ and cardiac index ( 3.27 vs. $\left.3.58 \mathrm{l} / \mathrm{min} / \mathrm{m}^{2}, \mathrm{p}<0.05\right)$. While noe administration was associated with a decrease in mean arterial pressure (93 vs. $88 \mathrm{mmHg}, \mathrm{p}<0.05$ ), an radrenaline dose and BFV did not change significantly, NIRS revealed a reduction in cerebral oxygenation, but $\mathrm{rSO}_{2}$ values remained within a healthy range in all patients (right 71 vs. $69 \%$, p<0.05; left 74 vs. $72 \%$, p<0.05).

Conclusions

In patients with $\mathrm{SAH}$, enteral nimodipine led to inconclusive changes in systemic haemodynamics. Although cerebral oxygenation as assessed by NIRS decreased after nimodipine administration, BFV remained stable.

Keywords: Cerebral blood flow velocity; Cerebral oxygenation; Nimodipine; Subarachnoid haemorrhage; Systemic haemodynamics 


\section{INTRODUCTION}

Subarachnoid haemorrhage after aneurysm rupture $(\mathrm{SAH})$ is associated with significant morbidity and mortality ${ }^{6}$. Delayed cerebral ischemia (DCI) is a clinical syndrome of focal neurological deficits that develop in one third of patients, and is a major cause of death and disability after $\mathrm{SAH}^{11,12)}$. Although further aspects like brain injury, inflammation and microthrombosis and their influence on DCI are currently being discussed ${ }^{13)}$, cerebral vasospasms (CVS) remain the most serious complication of $\mathrm{SAH}$, leading to DCI and infarction ${ }^{14)}$. CVS after SAH are described as narrowing of angiographically visible cerebral arteries ${ }^{6}$ and occur in $70 \%$ of patients with SAH after aneurysm rupture ${ }^{12}$. Typically, CVS occur between day 3 and 4 after aneurysm rupture, their peak is between day 7 to 10 and they resolve by day 14 to $2^{7,12)}$. Nimodipine remains the only approved medication to prevent $\mathrm{DCI}^{9)}$. As a lipophilic antagonist of calcium channels, nimodipine acts as a cerebrovascular vasodilator. In the largest randomized multicenter double-blind placebo-controlled study, nimodipine significantly reduced cerebral infarctions and poor outcome in patients with $\mathrm{SAH}^{8}$. According to the guidelines of the American Heart Association and the American Stroke Association, nimodipine should be administered orally to all patients with SAH (Class I, Level of Evidence $\mathrm{A})^{6}{ }^{6}$. Therefore, it is routinely administered (60 mg every four hours) from the time of SAH presentation through day $21^{6}$.

In clinical routine, transcranial Doppler (TCD) is a widely used tool to monitor for the development of SAH-induced CVS by assessing blood flow velocity (BFV) in the intracranial arteries ${ }^{6}$. Furthermore, near-infrared spectroscopy (NIRS) enables bedside brain monitoring by a non-invasive measurement of regional cerebral oxygen saturation $\left(\mathrm{rSO}_{2}\right.$ ) via absorption of near-infrared light of oxyhaemoglobin $(\mathrm{HbO})$, deoxyhaemoglobin $(\mathrm{Hb})$, and cytochrome-oxidase ${ }^{3,13,15,17,18)}$. Since there is still a lack of knowledge, we studied the systemic haemodynamic effects of nimodipine (as assessed by transpulmonary thermodilution with $\mathrm{PiCCO}^{\mathrm{TM}}$ technology) as well as its impact on $\mathrm{BFV}$ and $\mathrm{rSO}_{2}$ (as assessed by TCD and NIRS) in SAH patients.

\section{METHODS}

After approval from the institutional review board (Ethics Committee of the University of Witten/ Herdecke, number 48/2012, chair: Prof. Dr. med. P. Gaidzik, date of approval 2 September 2013), written individual informed consent was obtained for patients who presented at our hospital with SAH. In this prospective observational study, we included 18 adult critically ill patients with SAH between August 2014 and June 2018.
After SAH was diagnosed by computed tomography (CT), treatment was achieved by means of open surgical clipping or endovascular aneurysm obliteration. In all patients, an intracranial probe was inserted for continuous measurement of intracranial pressure (ICP). All patients were sedated and mechanically ventilated with continuous measurement of arterial blood pressure, heart rate, oxygen saturation and continuous recording of electrocardiogram. All patients enrolled underwent advanced hemodynamic monitoring with PiCCOTM technology (Pulsion, Maquet Getinge Group, Feldkirchen, Germany) due to poor clinical condition with haemodynamic instability. For the transpulmonary thermodilution measurement, a $15 \mathrm{ml}$ bolus of cold $0.9 \%$ saline was injected via a central venous catheter (V. cava superior). This procedure was repeated three times and the average was used to calculate the thermodilution parameters to calibrate the device for continuous pulse contour analysis. Regional cerebral oxygenation was detected by NIRS (INVOS ${ }^{\mathrm{TM}} 5100$ C, Somanetics, Minneapolis, MN, USA). Self-adhesive oximetry strips were applied bilaterally on the patient's forehead for continuous measurement of $\mathrm{rSO}_{2}$. TCD was performed to assess BFV of the intracranial arteries (middle cerebral artery (MCA) and anterior cerebral artery (ACA) using device (Multi-Dop ${ }^{\circledR}$, Singen, Germany).

Nimodipine was routinely administered (60 mg every four hours) via a gastric probe to all patients beginning from the time of SAH presentation. Measurements of systemic and cerebral haemodynamics as well as regional cerebral oxygenation were performed 30 minutes before and after the administration of nimodipine. This time point was chosen since, according to the technical information, nimodipine which after oral administration is detected in blood after 10-15 min with a maximum after about $40 \mathrm{~min}$. Each patient's examination and data collection were performed by the same examiner.

Data were tested for normal distribution by the Kolmogorov-Smirnov test. Data are expressed as mean \pm SD. The effect of nimodipine on systemic and cerebrovascular variables was compared using a paired t-test (Microsoft ${ }^{\circledR}$ Excel). P values less than 0.05 were considered as statistically significant.

\section{RESULTS}

Eighteen patients (mean age $59 \pm 11$ years, 11 females, 7 males) with SAH were included in this study (Table 1). Twelve aneurysms were treated with clip occlusion and five aneurysms were embolized with coils. In one case no bleeding source was found, hence no treatment was performed in this patient. After nimodipine administration a significant decrease in mean arterial pressure (MAP) (93 vs. $88 \mathrm{mmHg}, \mathrm{p}<0.05$ ) was observed together with a 
Table 1. Baseline characteristics of all patients

\begin{tabular}{|c|c|c|c|c|c|c|c|}
\hline Patient & Age (years) & Gender & Aneurysm localization & Hunt \& Hess grade & Treatment & SAPS II & SOFA \\
\hline 1 & 76 & male & $\mathrm{BA}$ & $\mathrm{V}$ & Coiling & 45 & 8 \\
\hline 2 & 76 & female & PICA & III & Coiling & 38 & 9 \\
\hline 3 & 54 & female & MCA/PICA & III & Clipping & 28 & 9 \\
\hline 4 & 62 & male & AComA & I & Clipping & 24 & 0 \\
\hline 5 & 44 & female & PCA & $\mathrm{V}$ & Coiling & 29 & 9 \\
\hline 6 & 56 & female & AComA & III & Clipping & 25 & 8 \\
\hline 7 & 51 & female & MCA & $\mathrm{V}$ & Clipping & 32 & 7 \\
\hline 8 & 50 & female & PICA & $\mathrm{V}$ & Coiling & 28 & 8 \\
\hline 9 & 50 & female & MCA & II & Clipping & 29 & 7 \\
\hline 10 & 50 & female & PCA & $\mathrm{I} / \mathrm{II}$ & Clipping & 23 & 7 \\
\hline 11 & 62 & female & MCA & IV & Clipping & 32 & 8 \\
\hline 12 & 80 & female & * & III & - & 46 & 7 \\
\hline 13 & 71 & male & ICA/PComA & III & Clipping & 46 & 9 \\
\hline 14 & 52 & male & ICA & IV & Clipping & 35 & 6 \\
\hline 15 & 53 & male & PComA & II & Coiling & 39 & 12 \\
\hline 16 & 55 & male & $\mathrm{BA}$ & III & Clipping & 31 & 8 \\
\hline 17 & 69 & female & $\mathrm{BA}$ & $\mathrm{V}$ & Clipping & 33 & 12 \\
\hline 18 & 54 & male & MCA & $\mathrm{V}$ & Clipping & 31 & 11 \\
\hline
\end{tabular}

BA: Basilar artery, PICA: Posterior inferior cerebellar artery, MCA: Middle cerebral artery, AComA: Anterior communicating artery, PCA: Posterior cerebral artery, ICA: Internal carotid artery, PComA: Posterior communicating artery, SAPS: Simplified acute physiology score, SOFA: Sequential organ failure assessment.

*Aneurysm was not detected by angiography.

concomitant increase in heart rate ( 75 vs. $79 / \mathrm{min}, \mathrm{p}<0.05)$. Furthermore, cardiac index $(\mathrm{CI})$ increased significantly (3.27 vs. 3.58 $1 / \mathrm{min} / \mathrm{m}^{2}, \mathrm{p}<0.05$ ) (Table 2). TCD showed normal and stable mean BFV in MCA and ACA before and after nimodipine administration. NIRS revealed a significant bifrontal reduction of $\mathrm{rSO}_{2}$ (right 71 vs. $69 \%, \mathrm{p}<0.05$; left 74 vs. $72 \%, \mathrm{p}<0.05$ ), yet staying within a physiological range among the study population (Table 3 ).

Elevated BFV of both MCA and ACA was seen in one patient, with values up to $200 \mathrm{~cm} / \mathrm{sec}$ (Fig. 1A and B). In this patient, nimodipine administration was associated with a further bilateral increase of BFV of MCA and ACA. While $\mathrm{rSO}_{2}$ values remained without significant change after nimodipine administration, NIRS detected a non-significantly lower right sided cerebral oxygen saturation (69 vs. $81 \%$ ) consistent with a considerably high BFV in MCA on the same side.

\section{DISCUSSION}

In patients with $\mathrm{SAH}$, we found that enteral nimodipine administration was associated with hypotension, an increase in heart rate and slightly higher noradrenaline requirements, while $\mathrm{CI}$ increased significantly. No significant changes in BFV in MCA and ACA were detected by TCD. Interestingly, NIRS revealed a decrease in frontal regional cerebral oxygenation, probably due to impaired cerebral autoregulation, however $\mathrm{rSO}_{2}$ values were within a physio-
Table 2. Systemic haemodynamics and vasopressor support before and 30 minutes after enteral administration of $60 \mathrm{mg}$ of nimodipine

\begin{tabular}{lccc}
\hline & Before & After & p-value \\
\hline Heart rate $(1 / \mathrm{min})$ & $75 \pm 19$ & $79 \pm 19$ & $<0.05$ \\
$\mathrm{MAP}(\mathrm{mmHg})$ & $93 \pm 14$ & $88 \pm 11$ & $<0.05$ \\
$\mathrm{CI}\left(\mathrm{l} / \mathrm{min} / \mathrm{m}^{2)}\right.$ & $3.27 \pm 0.67$ & $3.58 \pm 0.84$ & $<0.05$ \\
$\mathrm{CPP}(\mathrm{mmHg})$ & $83 \pm 17$ & $78 \pm 14$ & $<0.05$ \\
Norepinephrine $(\mu \mathrm{g} / \mathrm{kg} / \mathrm{min})$ & $0.25 \pm 0.20$ & $0.27 \pm 0.22$ & $<0.05$
\end{tabular}

MAP: Mean arterial pressure, CI: Cardiac index, CPP: Cerebral perfusion pressure.

Table 3. Variables of cerebral blood flow velocity and oxygenation before and 30 minutes after enteral administration of $60 \mathrm{mg}$ of nimodipine

\begin{tabular}{lccc}
\hline & Before & After & p-value \\
\hline BFV - MCA right $(\mathrm{cm} / \mathrm{sec})$ & $71 \pm 40$ & $71 \pm 40$ & 0.9 \\
BFV - MCA left $(\mathrm{cm} / \mathrm{sec})$ & $67 \pm 28$ & $68 \pm 35$ & 0.87 \\
$\mathrm{BFV}$ - ACA right $(\mathrm{cm} / \mathrm{sec})$ & $60 \pm 21$ & $60 \pm 33$ & 0.92 \\
$\mathrm{BFV}-$ ACA left $(\mathrm{cm} / \mathrm{sec})$ & $66 \pm 28$ & $69 \pm 35$ & 0.59 \\
$\mathrm{rSO}_{2}$ right $(\%)$ & $71 \pm 9$ & $69 \pm 10$ & $<0.05$ \\
$\mathrm{rSO}_{2}$ left $(\%)$ & $74 \pm 10$ & $72 \pm 8$ & $<0.05$
\end{tabular}

BFV: Blood flow velocity, MCA: Middle cerebral artery,ACA: Anterior cerebral artery, $\mathrm{rSO}_{2}$ : Regional cerebral oxygen saturation.

logical range in all patients.

In healthy subjects, nimodipine has shown to be effective in increasing cerebral perfusion in spite of its hypotensive effect. Similar 

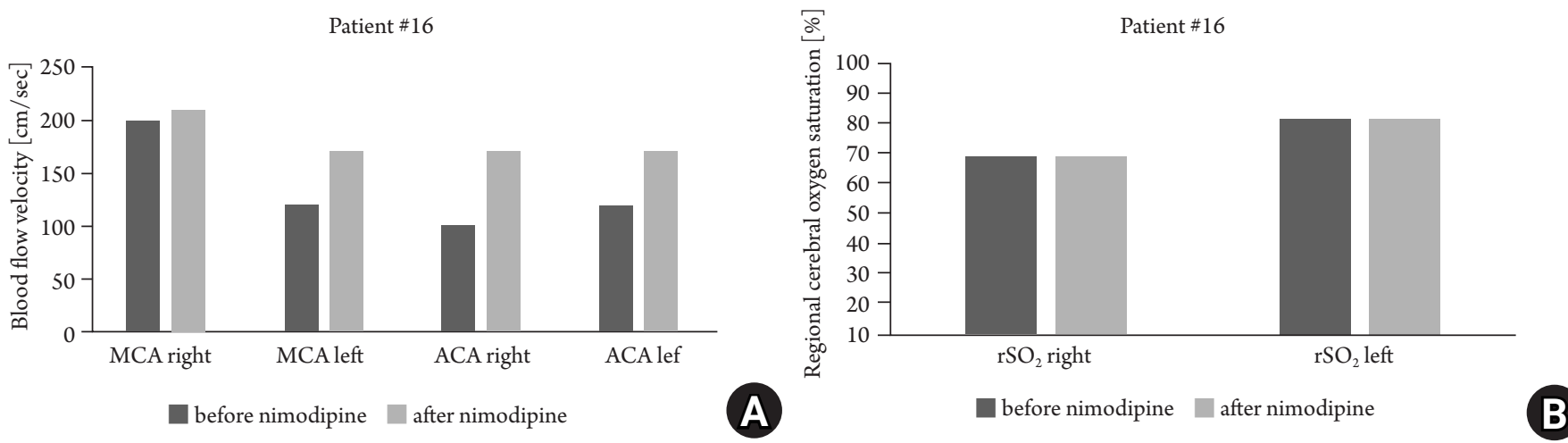

Fig. 1. (A) Blood flow velocity in middle cerebral artery (MCA) and anterior cerebral artery (ACA) before and 30 minutes after administration of $60 \mathrm{mg}$ of nimodipine in a patient with SAH. (B) Regional cerebral oxygen saturation $\mathrm{rSO}_{2}$ [\%] before and after nimodipine administration in a patient with SAH.

to our results, a decrease in arterial blood pressure with a concomitant increase in heart rate appeared without significant change of mean BFV in MCA. However, in contrast to our data, NIRS-derived regional oxygenation saturation improved after nimodipine administration ${ }^{4}$.

In patients with $\mathrm{SAH}$, the effect of nimodipine is ambiguous. Nimodipine seems to be unable to alter the incidence or severity of vasospasm ${ }^{6,12)}$, yet it reduces the risk of DCI and improves neurologic outcome after $\mathrm{SAH}^{12)}$. If clinically significant DCI occurs, arterial hypertension is recommended to improve cerebral perfu$\operatorname{sion}^{12)}$. While several studies report increased cerebral blood flow (CBF) due to nimodipine, few show reduced CBF linked to decreased MAP and $\mathrm{CPP}^{5,10,16)}$. In contrast, one retrospective study even revealed an enhanced risk of CVS with a higher need for vasopressor agents associated with nimodipine administration ${ }^{10)}$. Nimodipine is routinely used to prevent CVS, but its effect on patients with existing CVS might be risky. When nimodipine induces hypotension, an impaired autoregulation might lead to reduced cerebral perfusion with decreased $\mathrm{rSO}_{2}$ values in these patients. It remains unclear how many patients were indeed affected by CVS in the present study. TCD is widely used to consider CVS if mean $\mathrm{BFV}$ in MCA exceeds $120 \mathrm{~cm} / \mathrm{sec}^{10)}$. Overall, mean BFV in our patients remained within a physiological range. A pathologic increase of BFV only occurred in one patient in whom we found even further acceleration of $\mathrm{BFV}$ after nimodipine administration. In this case, $\mathrm{rSO}_{2}$ showed normal and stable values.

So far, no clear recommendation regarding the interpretation of NIRS values exists. Maslehaty et al. suggests $\mathrm{rSO}_{2}$ below $40 \%$ or a decrease by $25 \%$ from the baseline to be associated with DCI and neurological deficits ${ }^{13)}$. This did not appear in any of our patients. Either nimodipine administration did not lead to an improvement of cerebral perfusion and oxygenation as mean $\mathrm{BFV}$ and $\mathrm{rSO}_{2}$ remained within the normal range anyway, or the used methods were inadequate to detect a possible improvement.

When interpreting TCD- and NIRS-derived values, one should consider that TCD mainly assesses the MCA area, while bilaterally applied oximetry sensors on the forehead mainly involve the ACA territory and only partially covers the important MCA area ${ }^{13)}$. A modified application of the sensors should be considered. However, regardless of the applied location, near-infrared light only penetrates 2-2,5 cm into the head, hence NIRS only reflects the cortical region without assessing deeper regions of the brain ${ }^{14)}$. In addition, NIRS measurements can be tampered by factors like fever or sweat of the skin ${ }^{13)}$.

Though NIRS succeeded to detect improvement of $\mathrm{rSO}_{2}$ after intraarterial infusion of fasudil hydrochloride, a potent vasodilator, to a patient suffering from SAH-induced CVS14), its diagnostic value needs to be further investigated. Whether other methods as digital substraction angiography or perfusion $\mathrm{CT}$ would be able to detect an impact of nimodipine on $\mathrm{BFV}$ remains uncertain, too.

Our study has several limitations. As a lipophilic vasodilator, we expected nimodipine to cause cerebrovascular dilation with elevated $\mathrm{CBF}$ and $\mathrm{rSO}_{2}$. Due to its low bioavailability and in accordance with previous studies ${ }^{1,2,10)}$, it is conceivable that the gastric administration of nimodipine failed to attain therapeutic plasma concentrations needed to achieve the desired effect. However, in this clinical study, nimodipine plasma concentrations were not measured and hence a dose-effect relation cannot be analyzed. Furthermore, the sample size and the heterogeneity of the patient population are major limitations of this observational study.

\section{CONCLUSIONS}

In summary, enteral nimodipine is routinely used to prevent DCI, which can occur due to CVS in patients with SAH. In our study population, nimodipine showed inconclusive changes in sys- 
temic haemodynamics. BFV in MCA and ACA remained stable, not supporting the idea of cerebrovascular dilation following nimodipine administration. It remains unclear why $\mathrm{rSO}_{2}$ decreased bilaterally, when we assume the absence of CVS due to overall normal BFV in this study population. Further studies are required to conclude the cerebral effect of enteral nimodipine in patients with SAH.

\section{NOTES}

\section{Conflict of interest}

No potential conflict of interest relevant to this article was reported.

\section{REFERENCES}

1. Abboud T, Andresen H, Koeppen J, Czorlich P, Duehrsen L, Stenzig J, et al. Serum levels of nimodipine in enteral and parenteral administration in patients with aneurysmal subarachnoid hemorrhage. Acta Neurochir 2015;157:763-767.

2. Albanna W, Weiss M, Conzen C, Clusmann H, Schneider T, Reinsch M, et al. Systemic and Cerebral Concentration of Nimodipine During Established and Experimental Vasospasm Treatment. World Neurosurg 2017;102:459-465.

3. Bhatia R, Hampton T, Malde S, Kandala NB, Muammar M, Deasy N, et al. The application of near-infrared oximetry to cerebral monitoring during aneurysm embolization: a comparison with intraprocedural angiography.J Neurosurg Anesthesiol 2007;19:97-104.

4. Canova D, Roatta S, Micieli G, Bosone D. Cerebral oxygenation and haemodynamic effects induced by nimodipine in healthy subjects. Funct Neurol 2012;27:169-176.

5. Choi HA, Ko SB, Chen H, Gilmore E, Carpenter AM, Lee D, et al. Acute effects of nimodipine on cerebral vasculature and brain metabolism in high grade subarachnoid hemorrhage patients. Neurocrit Care 2012;16:363-367.

6. Rabinstein AA, Carhuapoma JR, Derdeyn CP, Dion J, Higashida RT, et al. Guidelines for the management of aneurysmal subarachnoid hemorrhage: a guideline for healthcare professionals from the American Heart Association/american Stroke Association. Stroke 2012;43:1711-1737.
7. Dorsch NW, King MT. A review of cerebral vasospasm in aneurysmal subarachnoid haemorrhage Part I: Incidence and effects. J Clin Neurosci 1994;1:19-26.

8. Grasso G, Alafaci C, Macdonald RL. Management of aneurysmal subarachnoid hemorrhage: State of the art and future perspectives. Surg Neurol Int 2017;8:11.

9. Highton D, Ghosh A, Tachtsidis I, Elwell C, Smith M. Analysis of slow wave oscillations in cerebral haemodynamics and metabolism following subarachnoid haemorrhage. Adv Exp Med Biol 2014;812:195-201.

10. Jaschinski U. [Intensive care treatment after aneurysmal subarachnoid hemorrhage]. Anaesthesist 2016;65:951-970.

11. Jane JA, Adams HP, Kongable GL. The International Cooperative Study on the Timing of Aneurysm Surgery. Part 1: Overall management results. J Neurosurg 1990;73:18-36.

12. Lawton MT, Vates GE. Subarachnoid Hemorrhage. N Engl J Med 2017;377:257-266.

13. Maslehaty H, Krause-Titz U, Petridis AK, Barth H, Mehdorn HM. Continuous measurement of cerebral oxygenation with near-infrared spectroscopy after spontaneous subarachnoid hemorrhage. ISRN Neurol 2012;2012:907187.

14. Mutoh T, Kobayashi S, Tamakawa N, Ishikawa T. Multichannel near-infrared spectroscopy as a tool for assisting intra-arterial fasudil therapy for diffuse vasospasm after subarachnoid hemorrhage. Surg Neurol Int 2011;2:68.

15. Mutoh T, Ishikawa T, Suzuki A, Yasui N. Continuous cardiac output and near-infrared spectroscopy monitoring to assist in management of symptomatic cerebral vasospasm after subarachnoid hemorrhage. Neurocrit Care 2010;13:331- 338.

16. Stiefel MF, Heuer GG, Abrahams JM, Bloom S, Smith MJ, Maloney-Wilensky E, et al. The effect of nimodipine on cerebral oxygenation in patients with poor-grade subarachnoid hemorrhage. J Neurosurg 2004;101:594-599.

17. Yokose N, Sakatani K, Murata Y, Awano T, Igarashi T, Nakamura $\mathrm{S}$, et al. Bedside assessment of cerebral vasospasms after subarachnoid hemorrhage by near infrared time-resolved spectroscopy. Adv Exp Med Biol 2010;662:505-511.

18. Zweifel C, Castellani G, Czosnyka M, Carrera E, Brady KM, Kirkpatrick PJ, et al. Continuous assessment of cerebral autoregulation with near-infrared spectroscopy in adults after subarachnoid hemorrhage. Stroke 2010;41:1963-1968. 